\title{
The differences in healthcare utilization for dental caries based on the implementation of water fluoridation in South Korea
}

\author{
Myung-Soo Cho ${ }^{1,2+}$, Kyu-Tae Han ${ }^{3,4 \dagger}$, Sohee Park ${ }^{5}$, Ki Tae Moon ${ }^{6}$ and Eun-Cheol Park ${ }^{4,7^{*}}$
}

\begin{abstract}
Background: There were some debates about the water fluoridation program in South Korea, even if the program had generally substantial effectiveness. Because the out-of-pocket expenditures for dental care were higher in South Korea than in other countries, an efficient solution was needed. Therefore, we examined the relationship between the implementation of water fluoridation and the utilization of dental care.

Methods: We used the National Health Insurance Service National Sample Cohort. In this study, data finally included 472,250 patients who were newly diagnosed with dental caries during 2003-2013. We performed survival analysis using cox proportional hazard model, negative binomial-regression, and regression analyses using generalized estimating equation models.

Results: There were $48.49 \%$ outpatient dental care visit during study period. Individuals with water fluoridation had a lower risk of dental care visits ( $\mathrm{HR}=0.949,95 \% \mathrm{Cl}=0.928-0.971)$. Among the individuals who experienced a dental care visit, those with water fluoridation program had a lower number of dental care visits $(\beta=-0.029)$, and the period of water fluoridation had an inverse association with the dental care expenditures.

Conclusion: The implementation of water fluoridation programs and these periods are associated with reducing the utilization of dental health care. Considering these positive impacts, healthcare professionals must consider preventive strategies for activating water fluoridation programs, such as changes in public perception and relations, for the effective management of dental care in South Korea.
\end{abstract}

Keywords: Water fluoridation, Dental caries, Healthcare expenditures, Public program

\section{Background}

Dental caries are the most common diseases related to oral health worldwide [1]. The insufficient management of oral health to prevent dental caries could cause several symptoms and large cost burdens associated with the treatment of dental caries. Naturally, many strategies for preventing dental caries were developed, such as the improvement of oral health behaviors, changes in dietary patterns, the brushing of teeth, the use of fluoride toothpaste, flu dental screenings, dental

\footnotetext{
* Correspondence: ecpark@yuhs.ac

${ }^{\dagger}$ Equal contributors

${ }^{4}$ Institute of Health Services Research, Yonsei University College of Medicine, Seoul, Republic of Korea

${ }^{7}$ Department of Preventive Medicine, Yonsei University College of Medicine,

Seoul, Republic of Korea

Full list of author information is available at the end of the article
}

scaling, and water fluoridation programs [2-4]. Recently, the oral health, especially that related to dental caries, of individuals has been generally improved compared with that in the past [5].

Water fluoridation is a program that introduces fluoride at an optimal level into drinking water to improve pubic dental health. It was first introduced in Grand Rapids in 1945 and was considered effective in the prevention of dental caries [6]. A previous report by the World Health Organization (WHO) suggested that the program could positively affect oral health, and the WHO recommended introducing water fluoridation in countries where such programs would be technically and culturally feasible [7]. Hence, many countries began to introduce water fluoridation. In the previous study which was conducted based on systematic review in UK, 
the water fluoridation had positive impact for reducing dental caries about $14 \%$ [8]. This program in New Zealand and Australia also made about positive outcomes in oral health related to dental caries $[9,10]$. The result in Japan also showed that positive effect too [11].

Since 1981, South Korea has implemented and gradually expanded a water fluoridation program. In 2002, the 32 regions among the $\sim 250$ regions in South Korea had implemented water fluoridation programs [12]. Since the late 1990s, some concerns about adverse effects of water fluoridation have been generally raised through media sources in South Korea. Each local government, with the support of voters, faces choices in its decision making for the implementation of water fluoridation. The expansion of water fluoridation thus faces difficulties, even if the program is effective in aspects of both quality of life and costs. Finally, only 17 regions implemented water fluoridation in 2013. Previous studies suggest, however, that there have been no significant adverse effects of introducing water fluoridation since the beginning of the 21st century [7, 8, 13].

Because of the effectiveness of water fluoridation and the continuous progress against dental caries in South Korea (age-adjusted prevalence rates of dental caries: $39.1 \%$ in $2007,31.4 \%$ in 2014), we assumed that water fluoridation programs could significantly affect the reduction of dental caries in South Korea [14]. Although some previous studies in South Korea examined the effectiveness of water fluoridation, there were no studies took the perspective of the whole nation in South Korea. Also, the cost burden, including out-of-pocket expenses, for dental care is one of the major causes of medical expenditures (2011: Organization for Economic Cooperation and Development [OECD] average $=55 \%$, South Korea $=84 \%$ ) [15]. Therefore, we examined the relationship between the implementation of water fluoridation and the utilization of dental care using national sample cohort data. Based on our findings, we expect that decision makers could make appropriate strategies for the management of oral health in South Korea.

\section{Methods}

\section{Study population}

The data used in this study was the National Health Insurance Service National Sample Cohort 2002-2013. It include a random sample of 1,025,340 individuals as about $2.2 \%$ of the overall South Korean in 2002. To represent total medical expenditure per year of South Korean within each of 1,476 strata (defined by age, sex, types of insurance coverage, and income level), this data was made through using probability sampling methods, and the model used proportional allocation. Thus, total medical claims of 1,025,340 individuals during 20022013 was included in this data. To examine the association between the implementation of water fluoridation program and the utilization of outpatient care for dental caries, represented by factors such as the frequency of outpatient visits and the medical expenditures by individuals, we only included patients who lived in non-metropolitan areas, because water fluoridation programs were rarely implemented in metropolitan areas (one metropolitan area in 2013). Then, we included only the patients who were newly diagnosed with dental caries (International Classification of Diseases [ICD]-10: K02) through outpatient care after 2003 (589,346 patients). We excluded those who were first diagnosed with dental caries before 2003 to ensure that only newly diagnosed patients were included. These patients were annually followed up. Finally, the data included 472,250 patients in 164 regions during 2003-2013 (average 9.12 years follow up). In addition, we added information about which regions had implemented water fluoridation from the Ministry of Health \& Welfare. Regional variables were obtained from the 'e-provincial indicators' which was collected by Statistics Korea. It contained the regional characteristics of about $250 \mathrm{Si}-\mathrm{Gun}-\mathrm{Gu}$ in South Korea. This data was used in this study for reflecting regional characteristics for each region where the patients with dental caries lived.

\section{Variables}

The outcome variables used in this study were based on information about healthcare utilization for dental caries within the study population. The first outcome variable used in this study was whether each patient visited to dental clinic due to dental caries through outpatient care during study period. Next, other outcome variables were used in this study were the frequencies of dental care visits and the cost of outpatient care as indicators of medical expenditures for only the patients who had experienced outpatient care for dental caries in each year.

The major variables of interest were whether or not each community implemented water fluoridation during the study period and how long the period of the program was. Based on 2013, the water fluoridation program was implemented in $13 \mathrm{Si}$-Gun-Gu among 164 non-metropolitan areas. In this study, the patients who lived in those regions were considered to live in regions where water fluoridation had been implemented. Additionally, to consider the effect of the time since the initial implementation the program, the time since the implementation of water fluoridation was also included in this study. We hypothesized that the implementation of water fluoridation and the time since the implementation in each region would affect the outpatient visits and medical expenditures by the patients.

The patients and regional variables were controlled in analyses for association between water fluoridation and 
the outcome variables. The patient variables were as follows: age, sex, income, type of insurance coverage, study year, dental care expenditures in the previous year, dental care visits in the previous year, dentofacial anomalies, and disorders of tooth development and eruption. The patients' age was categorized based on 10 years. The income was divided into deciles based on mean household income as follows: $\leq 10,11-20,21-30,31-40,41-50$, $51-60,61-70,71-80,81-90$, and $\geq 91 \%$. The types of insurance coverage were categorized based on the NHI criteria as: medical aid, National Health Insurance (NHI) employee insurance, or NHI self-employed insurance. First, medical aid was defined as individuals with income less than poverty level in South Korea or individuals with a physical or mental disability. They could receive the healthcare services as free or low copayment by government funds. And, the other individuals were defined as NHI beneficiaries. The NHI beneficiaries were categorized in employee or self-employed based on job status. If individuals included workers and employers in all workplaces, they were included to NHI employee insurance, and paid a premium of about $7 \%$ in their monthly income as withholding tax.The NHI self-employed insurance defined as individuals who did not fall into the NHI employee insurance group. They paid NHI premium based on their income, property, and living standard. Thus, this category could reflect the socio-economic status of each individual. We also included the number of pre-dental care visits, the pre-dental care cost, dentofacial anomalies (ICD-10: K07), and disorders of tooth development and eruption (ICD-10: K00) to adjust the severity of disease which could affect to the risk in dental caries in each patient. The number of pre-dental care visits was defined as the number of outpatient visits related to dental care during the previous year after the baseline year. The pre-dental care costs were calculated as the sum of the healthcare expenditures for dental care during the previous year after the baseline year. Dentofacial anomalies and disorders of tooth development and eruption were defined based on whether the patient was diagnosed with those symptoms as comorbidities in a specific year. The regional variables were the number of dentists per 1,000 people and the financial independence rate of the local government. The financial independence rate of the local government is an index of the finance utilization capacity of a local government with independent discretionary power. It was calculated as follows: (local taxes + non tax revenue)/budgets of local government $\times 100$.

\section{Statistical analysis}

First, we showed the frequencies of categorical variable and averages of continuous variable to examine the distribution of general characteristics at the baseline. We also performed $\chi^{2}$ tests and Cochran-Mantel-Haenszel tests to investigate the association between each categorical variables and visiting dental care by the implementation of water fluoridation as unit of person-years during study period. Next, we showed the averages and standard deviation of each continuous variable at the baseline and also performed a Mann-Whitney test, a Kruskal-Wallis test, and an analysis of covariance for continuous variable by the water fluoridation program during the study period. We also showed the Kaplan-Meier survival curve to compare the risk for visiting dental care by the water fluoridation. Finally, we performed a survival analysis using cox proportional hazard model to investigate whether patient visited to dental clinic due to dental caries during study period. Then, for the patients who had experienced outpatient care for dental caries during the study period, to examine the associations with the number of dental care visits and the dental care expenditures, we performed a multiple negative binomial regression, and regression analysis using a Generalized Estimating Equation (GEE) model with link logit regarding the overdispersion of outcome variables [16]. Additionally, we performed subgroup analyses according to age group or experience of predental care within each region to examine the difference of association related to water fluoridation. The statistical analyses in used this study were analyzed using SAS statistical software version 9.4.

\section{Results}

The data included 472,250 patients at baseline. Appendix 1 shows the general characteristics of the study population at baseline. The patients with water fluoridation were $10.40 \%$ of the total patients at baseline. The average follow-up time was 9.12 years during the study period.

Table 1 shows the associations among the patient and regional characteristics of baseline, the outpatient dental care visits for dental caries, and the presence of water fluoridation during the study period. The average percentage of patients that experienced an outpatient dental visit was $46.98 \%$ in regions with water fluoridation and $48.66 \%$ in regions without water fluoridation, respectively ( $p$-value $<.0001)$. The distributions of the other independent variables based on water fluoridation were similar among the groups. Females more frequently experienced outpatient dental care than males ( $p$-value $<.0001)$. In addition, younger or wealthier groups were generally more likely to make dental visits because of dental caries than other groups ( $p$-value $<.0001)$. Beneficiaries of NHI employee insurance more visited to dental care than individuals with other types of insurance $(p$-value $<.0001)$. 
Table 1 The association between baseline characteristics and dental care visit during study period by water fluoridation program

\begin{tabular}{|c|c|c|c|c|c|c|c|c|c|c|c|}
\hline \multirow[t]{4}{*}{ Variables } & \multicolumn{10}{|c|}{ Water flouridation } & \multirow[t]{4}{*}{$P$-value } \\
\hline & \multicolumn{5}{|l|}{ Yes } & \multicolumn{5}{|l|}{ No } & \\
\hline & \multicolumn{2}{|l|}{ Visit } & \multicolumn{2}{|l|}{ Non-visit } & \multirow[t]{2}{*}{$P$-value } & & & & \multirow[t]{2}{*}{$P$-value } & \\
\hline & N/Mean & $\% / S D$ & N/Mean & $\% / S D$ & & N/Mean & $\% / S D$ & N/Mean & $\% / S D$ & & \\
\hline \multicolumn{12}{|l|}{ Regional variables } \\
\hline $\begin{array}{l}\text { Period from introduction of } \\
\text { water fluoridation }\end{array}$ & 6.40 & \pm 4.67 & 6.64 & \pm 4.74 & $<.0001$ & - & - & - & - & - & - \\
\hline $\begin{array}{l}\text { Number of dentists per 1,000 } \\
\text { residents }\end{array}$ & 0.29 & \pm 0.15 & 0.29 & \pm 0.15 & $<.0001$ & 0.28 & \pm 0.12 & 0.27 & \pm 0.12 & $<.0001$ & 0.0021 \\
\hline $\begin{array}{l}\text { Financial independence rate } \\
\text { of local government }\end{array}$ & 46.48 & \pm 23.11 & 47.56 & \pm 23.36 & $<.0001$ & 51.77 & \pm 24.63 & 50.36 & \pm 24.45 & $<.0001$ & $<.0001$ \\
\hline \multicolumn{12}{|l|}{ Individual variables } \\
\hline \multicolumn{12}{|l|}{ Sex } \\
\hline Male & 10,934 & 44.14 & 13,837 & 55.86 & $<.0001$ & 98,496 & 45.88 & 116,178 & 54.12 & $<.0001$ & $<.0001$ \\
\hline Female & 12,142 & 49.86 & 12,209 & 50.14 & & 107,402 & 51.52 & 101,052 & 48.48 & & \\
\hline \multicolumn{12}{|l|}{ Age (Years) } \\
\hline-19 & 8,880 & 59.43 & 6,063 & 40.57 & $<.0001$ & 78,779 & 61.62 & 49,071 & 38.38 & $<.0001$ & $<.0001$ \\
\hline $20-29$ & 3,546 & 51.14 & 3,388 & 48.86 & & 31,139 & 51.96 & 28,790 & 48.04 & & \\
\hline $30-39$ & 3,663 & 43.75 & 4,710 & 56.25 & & 32,436 & 45.26 & 39,233 & 54.74 & & \\
\hline $40-49$ & 3,290 & 43.90 & 4,205 & 56.10 & & 29,275 & 44.93 & 35,884 & 55.07 & & \\
\hline $50-59$ & 1,872 & 43.30 & 2,451 & 56.70 & & 16,961 & 44.98 & 20,743 & 55.02 & & \\
\hline $60-69$ & 1,366 & 34.45 & 2,599 & 65.55 & & 12,956 & 37.25 & 21,824 & 62.75 & & \\
\hline$<70$ & 459 & 14.86 & 2,630 & 85.14 & & 4,352 & 16.71 & 21,685 & 83.29 & & \\
\hline \multicolumn{12}{|l|}{ Income (percentile) } \\
\hline$-10 \%$ (low) & 1,911 & 35.95 & 3,405 & 64.05 & $<.0001$ & 15,930 & 35.78 & 28,588 & 64.22 & $<.0001$ & $<.0001$ \\
\hline $11-20 \%$ & 1,404 & 43.58 & 1,818 & 56.42 & & 12,124 & 44.91 & 14,874 & 55.09 & & \\
\hline $21-30 \%$ & 1,705 & 44.68 & 2,111 & 55.32 & & 14,157 & 46.55 & 16,253 & 53.45 & & \\
\hline $31-40 \%$ & 2,014 & 45.56 & 2,407 & 54.44 & & 16,787 & 46.70 & 19,163 & 53.30 & & \\
\hline $41-50 \%$ & 2,289 & 46.19 & 2,667 & 53.81 & & 19,872 & 48.74 & 20,899 & 51.26 & & \\
\hline $51-60 \%$ & 2,518 & 47.58 & 2,774 & 52.42 & & 21,769 & 49.95 & 21,811 & 50.05 & & \\
\hline $61-70 \%$ & 2,818 & 49.68 & 2,854 & 50.32 & & 24,962 & 50.83 & 24,148 & 49.17 & & \\
\hline $71-80 \%$ & 2,988 & 50.50 & 2,929 & 49.50 & & 27,172 & 52.05 & 25,032 & 47.95 & & \\
\hline $81-90 \%$ & 2,840 & 50.87 & 2,743 & 49.13 & & 28,038 & 52.66 & 25,206 & 47.34 & & \\
\hline + $91 \%$ (high) & 2,589 & 52.55 & 2,338 & 47.45 & & 25,087 & 54.13 & 21,256 & 45.87 & & \\
\hline \multicolumn{12}{|l|}{ Types of insurance coverage } \\
\hline Medical Aid & 449 & 22.90 & 1,512 & 77.10 & $<.0001$ & 3,868 & 23.24 & 12,778 & 76.76 & $<.0001$ & $<.0001$ \\
\hline $\mathrm{NHI}$, self-employed insured & 10,619 & 46.96 & 11,995 & 53.04 & & 92,100 & 48.14 & 99,217 & 51.86 & & \\
\hline $\mathrm{NHI}$, employee insured & 12,008 & 48.92 & 12,539 & 51.08 & & 109,930 & 51.09 & 105,235 & 48.91 & & \\
\hline \multicolumn{12}{|l|}{ Year of baseline } \\
\hline 2003 & 20,564 & 47.40 & 22,819 & 52.60 & $<.0001$ & 180,731 & 49.01 & 187,996 & 50.99 & $<.0001$ & $<.0001$ \\
\hline 2004 & 661 & 55.22 & 536 & 44.78 & & 5,917 & 58.95 & 4,120 & 41.05 & & \\
\hline 2005 & 412 & 61.40 & 259 & 38.60 & & 3,783 & 65.57 & 1,986 & 34.43 & & \\
\hline 2006 & 346 & 58.25 & 248 & 41.75 & & 3,595 & 58.85 & 2,514 & 41.15 & & \\
\hline 2007 & 271 & 51.82 & 252 & 48.18 & & 3,056 & 57.40 & 2,268 & 42.60 & & \\
\hline 2008 & 272 & 47.97 & 295 & 52.03 & & 2,897 & 57.84 & 2,112 & 42.16 & & \\
\hline 2009 & 221 & 42.91 & 294 & 57.09 & & 2,276 & 49.04 & 2,365 & 50.96 & & \\
\hline
\end{tabular}


Table 1 The association between baseline characteristics and dental care visit during study period by water fluoridation program (Continued)

\begin{tabular}{|c|c|c|c|c|c|c|c|c|c|c|c|}
\hline 2010 & 170 & 37.53 & 283 & 62.47 & & 1,808 & 41.23 & 2,577 & 58.77 & & \\
\hline 2011 & 96 & 24.55 & 295 & 75.45 & & 1,093 & 26.17 & 3,083 & 73.83 & & \\
\hline 2012 & 44 & 10.21 & 387 & 89.79 & & 563 & 12.55 & 3,924 & 87.45 & & \\
\hline 2013 & 19 & 4.79 & 378 & 95.21 & & 179 & 4.01 & 4,285 & 95.99 & & \\
\hline \multicolumn{12}{|c|}{ Dentofacial anomalies } \\
\hline Yes & 13 & 100.00 & 0 & 0.00 & 0.0001 & 70 & 100.00 & 0 & 0.00 & $<.0001$ & $<.0001$ \\
\hline No & 23,063 & 46.96 & 26,046 & 53.04 & & 205,828 & 48.65 & 217,230 & 51.35 & & \\
\hline \multicolumn{12}{|c|}{ Disorders of toothe development and eruption } \\
\hline Yes & 89 & 100.00 & 0 & 0.00 & $<.0001$ & 792 & 100.00 & 0 & 0.00 & $<.0001$ & $<.0001$ \\
\hline No & 22,987 & 46.88 & 26,046 & 53.12 & & 205,106 & 48.56 & 217,230 & 51.44 & & \\
\hline Total & 23,07 & 46.98 & 26,046 & 53.02 & & 205,898 & 48.66 & 217,230 & 51.34 & & $<.0001$ \\
\hline
\end{tabular}

†The results of a Cochran-Mantel-Haenszel test to investigate the differences of distribution for categorical variables based on water fluoridation

Appendix 2 shows the Kaplan-Meier survival curve for time to first diagnosis of dental caries by the implementation water fluoridation. Individuals in regions with water fluoridation program was less likely to visit dental care than individuals in regions without program ( $p$-value for log-rank test <.0001).

Table 2 shows the distribution based on water fluoridation of the average values and the standard deviation for dental care visits or cost per year among only the patients who made dental visits because of dental caries. The average number of dental care visits and the costs were lower among patients with water fluoridation than among those without the program $(p$-value $<.0001)$.

Table 3 shows the results of the survival analysis using cox proportional hazard model, negative binomial regression, and regression analysis for dental care expenditures, represented by the number of visits or the costs, among only the patients that experienced a dental visit. Patient with water fluoridation program had higher risk in the dental care visit during study period (Yes $=$ HR: 0.949, $95 \%$ CI: 0.928-0.971, $p$-value <.0001). Female and younger people was had more tend to dental care visits due to dental caries ( $p$-value $<.0001)$. By the economic status, people with higher income or employee insured NHI had high risk in dental care visits than other groups $(p$-value $<.0001)$. In addition, people with dental comorbidities was also more visited in dental care ( $p$-value <.0001).

The results of the regression analysis for dental care expenditures showed that patients in regions that implemented a water fluoridation program had a lower number of dental visits or lower costs, although the results about dental care cost was not statistically significant (number of dental visits: $\beta=-0.029, p$-value $=$ 0.0431 ; dental care costs: $\beta=-0.008, p$-value $=0.7097$ ). In addition, patients in regions with more dentists made less dental visits and spent less on dental care. On the other hand, the financial independence rate in each region had an inverse association with the risk of dental visits $(p$-value $<.05)$. For the patient characteristics, males had an inverse association with the number of dental visits and costs $(p$-value $<.0001)$. On the other hand, beneficiaries of Medical Aid or self-employed NHI had a lower number of dental visits and lower costs than beneficiaries of employee NHI ( $p$-value $<.05)$. Expenditures related to pre-dental care or comorbidity had a positive association with the outcome variables $(p$-value $<.001)$.

We performed subgroup analyses for the survival analysis using cox proportional hazard model, negative binomial regression, and regression analyses using GEE models to investigate the relationship between water fluoridation and the outcome variables based on experience of pre-dental care visits (visit or no visit), income $(-50 \%$ and $+51 \%)$, and age group ( $\leq 19$ years, $20-39$ years, $40-59$ years, $\geq 60$ years). The associations between the presence of a water fluoridation program and the number of dental visits was greater for patients who had not experienced a pre-dental care visit in the previous year than for patients who had experienced a predental care visit in the previous year (with, water fluoridation $=\beta: 0.043, p$-value $=0.2901$; without, water fluoridation $=\beta: \quad-0.031, \quad p$-value $=0.0424) . \quad$ By age group, the inverse associations between water fluoridation and outcome variables were greater in the elderly population than in the younger population. In the younger groups, the inverse associations with dental care expenditures were analyzed based on period from implementation of water fluoridation (Table 4).

\section{Discussion}

To effectively manage oral health, and particularly patients with dental caries in South Korea, the South Korean government has introduced water fluoridation 
Table 2 The averages and standard deviations of dental care visits and costs for patients who visited dental care through outpatient caret

\begin{tabular}{|c|c|c|c|c|c|c|c|c|c|c|c|c|c|c|}
\hline \multirow[t]{4}{*}{ Variables } & \multicolumn{6}{|c|}{ Water flouridation } & \multirow[t]{4}{*}{$P$-value } & \multicolumn{6}{|c|}{ Water flouridation } & \multirow[t]{4}{*}{$P$-valuet } \\
\hline & \multicolumn{3}{|l|}{ Yes } & \multicolumn{3}{|l|}{ No } & & \multicolumn{3}{|l|}{ Yes } & \multicolumn{3}{|l|}{ No } & \\
\hline & \multicolumn{2}{|c|}{ Number of dental care visits } & \multirow[t]{2}{*}{$P$-value } & \multicolumn{2}{|c|}{ Number of dental care visits } & \multirow[t]{2}{*}{$P$-value } & & \multicolumn{2}{|c|}{ Dental care costs (KRW) } & \multirow[t]{2}{*}{$P$-value } & \multicolumn{2}{|c|}{ Dental care costs (KRW) } & \multirow[t]{2}{*}{$P$-value } & \\
\hline & Mean & SD & & Mean & SD & & & Mean & SD & & Mean & SD & & \\
\hline \multicolumn{15}{|l|}{ Individual variables } \\
\hline \multicolumn{15}{|l|}{ Sex } \\
\hline Male & 0.28 & \pm 0.75 & $<.0001$ & 0.30 & \pm 0.79 & $<.0001$ & 0.9079 & 8485.36 & $\pm 26,233.38$ & 0.0167 & 8758.21 & $\pm 27,035.59$ & $<.0001$ & 0.6855 \\
\hline Female & 0.29 & \pm 0.77 & & 0.30 & \pm 0.81 & & & 8567.78 & $\pm 26,234.69$ & & 8790.68 & $\pm 26,725.42$ & & \\
\hline \multicolumn{15}{|l|}{ Age (Years) } \\
\hline-19 & 0.38 & \pm 0.90 & $<.0001$ & 0.42 & \pm 0.95 & $<.0001$ & $<.0001$ & 12358.18 & $\pm 32,803.66$ & $<.0001$ & 12790.26 & $\pm 33,474.42$ & $<.0001$ & 0.2527 \\
\hline $20-29$ & 0.26 & \pm 0.72 & & 0.27 & \pm 0.76 & & & 7478.72 & $\pm 24,358.22$ & & 7636.75 & $\pm 24,858.38$ & & \\
\hline $30-39$ & 0.22 & \pm 0.65 & & 0.23 & \pm 0.67 & & & 6137.69 & $\pm 20,783.50$ & & 6353.85 & $\pm 21,809.12$ & & \\
\hline $40-49$ & 0.22 & \pm 0.63 & & 0.24 & \pm 0.68 & & & 6118.31 & $\pm 20,360.00$ & & 6575.26 & $\pm 22,017.51$ & & \\
\hline $50-59$ & 0.25 & \pm 0.69 & & 0.24 & \pm 0.68 & & & 7203.43 & $\pm 22,910.40$ & & 7305.94 & $\pm 23,947.33$ & & \\
\hline $60-69$ & 0.26 & \pm 0.77 & & 0.27 & \pm 0.75 & & & 7729.45 & $\pm 25,167.92$ & & 7685.72 & $\pm 24,254.82$ & & \\
\hline$<70$ & 0.26 & \pm 0.76 & & 0.27 & \pm 0.76 & & & 7378.33 & $\pm 23,917.36$ & & 7392.36 & $\pm 23,288.21$ & & \\
\hline \multicolumn{15}{|l|}{ Income (percentile) } \\
\hline$-10 \%$ (low) & 0.25 & \pm 0.74 & 0.0003 & 0.27 & \pm 0.81 & $<.0001$ & 0.2805 & 7745.45 & $\pm 25,837.11$ & 0.0003 & 7969.37 & $\pm 26,342.94$ & $<.0001$ & 0.0164 \\
\hline $11-20 \%$ & 0.28 & \pm 0.78 & & 0.29 & \pm 0.81 & & & 8170.88 & $\pm 26,355.37$ & & 8539.98 & $\pm 26,535.13$ & & \\
\hline $21-30 \%$ & 0.28 & \pm 0.79 & & 0.29 & \pm 0.80 & & & 8300.89 & $\pm 26,029.00$ & & 8530.71 & $\pm 26,384.56$ & & \\
\hline $31-40 \%$ & 0.26 & \pm 0.70 & & 0.29 & \pm 0.80 & & & 7579.31 & $\pm 24,080.07$ & & 8585.70 & $\pm 27,179.61$ & & \\
\hline $41-50 \%$ & 0.28 & \pm 0.76 & & 0.30 & \pm 0.80 & & & 8685.57 & $\pm 27,015.42$ & & 8710.41 & $\pm 26,762.40$ & & \\
\hline $51-60 \%$ & 0.29 & \pm 0.77 & & 0.30 & \pm 0.80 & & & 8869.81 & $\pm 27,295.76$ & & 8910.14 & $\pm 27,358.22$ & & \\
\hline $61-70 \%$ & 0.30 & \pm 0.78 & & 0.31 & \pm 0.81 & & & 8887.83 & $\pm 26,420.46$ & & 9195.21 & $\pm 27,617.53$ & & \\
\hline $71-80 \%$ & 0.29 & \pm 0.77 & & 0.32 & \pm 0.81 & & & 8868.81 & $\pm 26,543.89$ & & 9330.15 & $\pm 27,794.34$ & & \\
\hline $81-90 \%$ & 0.30 & \pm 0.79 & & 0.32 & \pm 0.80 & & & 9128.50 & $\pm 27,290.42$ & & 9128.64 & $\pm 27,025.66$ & & \\
\hline + $91 \%$ (high) & 0.28 & \pm 0.72 & & 0.30 & \pm 0.76 & & & 8348.43 & $\pm 24,746.39$ & & 8392.44 & $\pm 25,500.98$ & & \\
\hline \multicolumn{15}{|l|}{ Types of insurance coverage } \\
\hline Medical Aid & 0.17 & \pm 0.65 & $<.0001$ & 0.19 & \pm 0.73 & $<.0001$ & 0.9777 & 5463.02 & $\pm 24,295.15$ & $<.0001$ & 5569.94 & $\pm 23,732.00$ & $<.0001$ & 0.6780 \\
\hline $\begin{array}{l}\mathrm{NHI} \text {, self-employed } \\
\text { insured }\end{array}$ & 0.27 & \pm 0.75 & & 0.29 & \pm 0.79 & & & 8349.77 & $\pm 25,975.77$ & & 8657.48 & $\pm 26,710.94$ & & \\
\hline $\mathrm{NHI}$, employee insured & 0.29 & \pm 0.77 & & 0.31 & \pm 0.81 & & & 8768.09 & $\pm 26,464.81$ & & 8971.88 & $\pm 27,079.50$ & & \\
\hline
\end{tabular}


Table 2 The averages and standard deviations of dental care visits and costs for patients who visited dental care through outpatient caret (Continued)

\begin{tabular}{|c|c|c|c|c|c|c|c|c|c|c|c|c|c|c|}
\hline \multicolumn{15}{|c|}{ Index year } \\
\hline 2003 & 0.25 & \pm 0.61 & $<.0001$ & 0.25 & \pm 0.63 & $<.0001$ & $<.0001$ & 8034.83 & $\pm 24,159.19$ & $<.0001$ & 8080.83 & $\pm 24,641.98$ & $<.0001$ & 0.0003 \\
\hline 2004 & 0.25 & \pm 0.63 & & 0.25 & \pm 0.62 & & & 8382.93 & $\pm 26,010.94$ & & 8009.35 & $\pm 24,967.53$ & & \\
\hline 2005 & 0.25 & \pm 0.61 & & 0.25 & \pm 0.63 & & & 8297.59 & $\pm 25,261.94$ & & 8270.24 & $\pm 25,774.14$ & & \\
\hline 2006 & 0.25 & \pm 0.63 & & 0.25 & \pm 0.63 & & & 8283.21 & $\pm 25,874.99$ & & 8310.02 & $\pm 26,017.55$ & & \\
\hline 2007 & 0.29 & \pm 0.78 & & 0.30 & \pm 0.82 & & & 8302.10 & $\pm 25,361.18$ & & 8447.74 & $\pm 25,742.34$ & & \\
\hline 2008 & 0.33 & \pm 0.90 & & 0.34 & \pm 0.93 & & & 8527.55 & $\pm 25,215.56$ & & 8613.98 & $\pm 25,737.46$ & & \\
\hline 2009 & 0.32 & \pm 0.87 & & 0.34 & \pm 0.91 & & & 8484.86 & $\pm 25,234.96$ & & 8797.15 & $\pm 26,417.00$ & & \\
\hline 2010 & 0.30 & \pm 0.83 & & 0.32 & \pm 0.88 & & & 8363.59 & $\pm 26,338.25$ & & 8692.93 & $\pm 26,825.06$ & & \\
\hline 2011 & 0.31 & \pm 0.84 & & 0.34 & \pm 0.90 & & & 8821.34 & $\pm 27,704.86$ & & 9519.33 & $\pm 28,487.64$ & & \\
\hline 2012 & 0.30 & \pm 0.83 & & 0.33 & \pm 0.88 & & & 9062.00 & $\pm 28,211.94$ & & 9642.10 & $\pm 29,482.37$ & & \\
\hline 2013 & 0.29 & \pm 0.79 & & 0.33 & \pm 0.87 & & & 9443.44 & $\pm 29,389.48$ & & 10376.20 & $\pm 31,264.12$ & & \\
\hline \multicolumn{15}{|c|}{ Dentofacial anomalies } \\
\hline Yes & 1.75 & \pm 1.32 & $<.0001$ & 1.91 & \pm 1.42 & $<.0001$ & 0.0774 & 60991.70 & $\pm 60,760.65$ & $<.0001$ & 68584.30 & $\pm 65,057.42$ & $<.0001$ & 0.0088 \\
\hline No & 0.28 & \pm 0.76 & & 0.30 & \pm 0.80 & & & 8503.58 & $\pm 26,182.17$ & & 8742.72 & $\pm 26,802.56$ & & \\
\hline \multicolumn{15}{|c|}{ Disorders of toothe development and eruption } \\
\hline Yes & 1.89 & \pm 1.43 & $<.0001$ & 1.95 & \pm 1.39 & $<.0001$ & 0.1953 & 70958.60 & $\pm 56,207.42$ & $<.0001$ & 71028.40 & $\pm 54,669.15$ & $<.0001$ & 0.8636 \\
\hline No & 0.28 & \pm 0.75 & & 0.29 & \pm 0.79 & & & 8287.70 & $\pm 25,760.74$ & & 8526.49 & $\pm 26,412.60$ & & \\
\hline Total & 0.28 & \pm 0.76 & & 0.30 & \pm 0.80 & & $<.0001$ & 8528.51 & $\pm 26,234.03$ & & 8775.16 & $\pm 26,874.15$ & & $<.0001$ \\
\hline
\end{tabular}

$\uparrow$ The results of analysis of covariance to investigate the differences of distribution for continuous variables based on water fluoridation 
Table 3 The results of survival analysis, negative binomial regression, and regression for dental care visits and expenditures including both patient and regional characteristics

\begin{tabular}{|c|c|c|c|c|c|c|c|c|c|c|}
\hline \multirow[t]{3}{*}{ Variables } & \multirow{2}{*}{\multicolumn{4}{|c|}{ Dental care visits ${ }^{a}$}} & \multicolumn{6}{|c|}{ Only patient with dental care visit } \\
\hline & & & & & \multicolumn{3}{|c|}{ Number of dental care visits ${ }^{b}$} & \multicolumn{3}{|c|}{ Dental care costs $(\mathrm{KRW})^{\mathrm{C}}$} \\
\hline & $\overline{\mathrm{HR}}$ & \multicolumn{2}{|c|}{$95 \% \mathrm{Cl}$} & $P$-value & $\bar{\beta}$ & SE & $P$-value & $\beta$ & SE & $P$-value \\
\hline \multicolumn{11}{|l|}{ Regional variables } \\
\hline \multicolumn{11}{|l|}{ Water fluoridation } \\
\hline Yes & 0.949 & 0.928 & 0.971 & $<.0001$ & -0.029 & 0.014 & 0.0431 & 0.008 & 0.021 & 0.7097 \\
\hline No & 1.000 & - & - & - & Ref & - & - & Ref & - & - \\
\hline $\begin{array}{l}\text { Period from implementation of water } \\
\text { fluoridation }\end{array}$ & 0.998 & 0.995 & 1.001 & 0.2302 & -0.003 & 0.001 & 0.0177 & -0.006 & 0.002 & 0.0024 \\
\hline Number of dentists per 1,000 residents & 1.007 & 0.975 & 1.041 & 0.6700 & -0.045 & 0.016 & 0.0068 & -0.026 & 0.024 & 0.2812 \\
\hline $\begin{array}{l}\text { Financial independence rate of local } \\
\text { government }\end{array}$ & 0.999 & 0.999 & 0.999 & $<.0001$ & -0.003 & 0.000 & $<.0001$ & -0.003 & 0.000 & $<.0001$ \\
\hline \multicolumn{11}{|l|}{ Individual variables } \\
\hline \multicolumn{11}{|l|}{ Sex } \\
\hline Male & 0.795 & 0.788 & 0.801 & $<.0001$ & -0.051 & 0.004 & $<.0001$ & -0.071 & 0.005 & $<.0001$ \\
\hline Female & 1.000 & - & - & - & Ref & - & - & Ref & - & - \\
\hline \multicolumn{11}{|l|}{ Age (Years) } \\
\hline-19 & 4.835 & 4.695 & 4.979 & $<.0001$ & 0.408 & 0.010 & $<.0001$ & 0.662 & 0.013 & $<.0001$ \\
\hline $20-29$ & 3.069 & 2.977 & 3.163 & $<.0001$ & 0.064 & 0.011 & $<.0001$ & 0.020 & 0.014 & 0.1451 \\
\hline $30-39$ & 2.466 & 2.392 & 2.542 & $<.0001$ & -0.073 & 0.011 & $<.0001$ & -0.134 & 0.014 & $<.0001$ \\
\hline $40-49$ & 2.502 & 2.427 & 2.579 & $<.0001$ & -0.069 & 0.011 & $<.0001$ & -0.101 & 0.013 & $<.0001$ \\
\hline $50-59$ & 2.531 & 2.452 & 2.613 & $<.0001$ & -0.022 & 0.011 & 0.0487 & -0.030 & 0.014 & 0.0316 \\
\hline $60-69$ & 2.044 & 1.978 & 2.112 & $<.0001$ & 0.033 & 0.012 & 0.0045 & 0.055 & 0.016 & 0.0004 \\
\hline$<70$ & 1.000 & - & - & - & Ref & - & - & Ref & - & - \\
\hline \multicolumn{11}{|l|}{ Income (percentile) } \\
\hline$-10 \%$ (low) & 0.751 & 0.736 & 0.767 & $<.0001$ & 0.003 & 0.009 & 0.7721 & -0.085 & 0.013 & $<.0001$ \\
\hline $11-20 \%$ & 0.751 & 0.736 & 0.767 & $<.0001$ & -0.004 & 0.009 & 0.6797 & -0.094 & 0.013 & $<.0001$ \\
\hline $21-30 \%$ & 0.762 & 0.747 & 0.777 & $<.0001$ & -0.004 & 0.009 & 0.6740 & -0.099 & 0.013 & $<.0001$ \\
\hline $31-40 \%$ & 0.770 & 0.756 & 0.784 & $<.0001$ & -0.026 & 0.008 & 0.0018 & -0.121 & 0.012 & $<.0001$ \\
\hline $41-50 \%$ & 0.805 & 0.790 & 0.819 & $<.0001$ & 0.006 & 0.008 & 0.4173 & -0.069 & 0.012 & $<.0001$ \\
\hline $51-60 \%$ & 0.835 & 0.821 & 0.850 & $<.0001$ & 0.011 & 0.008 & 0.1536 & -0.045 & 0.012 & 0.0001 \\
\hline $61-70 \%$ & 0.880 & 0.866 & 0.895 & $<.0001$ & 0.028 & 0.007 & 0.0002 & -0.007 & 0.011 & 0.5510 \\
\hline $71-80 \%$ & 0.916 & 0.901 & 0.931 & $<.0001$ & 0.030 & 0.007 & $<.0001$ & 0.025 & 0.011 & 0.0267 \\
\hline $81-90 \%$ & 0.943 & 0.928 & 0.959 & $<.0001$ & 0.023 & 0.007 & 0.0007 & 0.025 & 0.011 & 0.0190 \\
\hline +91\% (high) & 1.000 & - & - & - & Ref & - & - & Ref & - & - \\
\hline \multicolumn{11}{|l|}{ Types of insurance coverage } \\
\hline Medical Aid & 0.410 & 0.396 & 0.425 & $<.0001$ & -0.573 & 0.018 & $<.0001$ & -0.956 & 0.017 & $<.0001$ \\
\hline $\mathrm{NHI}$, self-employed insured & 0.872 & 0.865 & 0.879 & $<.0001$ & -0.033 & 0.004 & $<.0001$ & -0.097 & 0.006 & $<.0001$ \\
\hline $\mathrm{NHI}$, employee insured & 1.000 & - & - & - & Ref & - & - & Ref & - & - \\
\hline \multicolumn{11}{|l|}{ Year of baseline/Index year } \\
\hline 2003 & 1.000 & - & - & - & Ref & - & - & Ref & - & - \\
\hline 2004 & 1.052 & 1.026 & 1.079 & $<.0001$ & -0.015 & 0.003 & $<.0001$ & -0.126 & 0.013 & $<.0001$ \\
\hline 2005 & 1.126 & 1.092 & 1.162 & $<.0001$ & -0.018 & 0.003 & $<.0001$ & -0.102 & 0.013 & $<.0001$ \\
\hline 2006 & 1.124 & 1.089 & 1.160 & $<.0001$ & -0.017 & 0.003 & $<.0001$ & -0.108 & 0.013 & $<.0001$ \\
\hline 2007 & 1.143 & 1.104 & 1.183 & $<.0001$ & 0.128 & 0.004 & $<.0001$ & -0.037 & 0.013 & 0.0037 \\
\hline
\end{tabular}


Table 3 The results of survival analysis, negative binomial regression, and regression for dental care visits and expenditures including both patient and regional characteristics (Continued)

\begin{tabular}{|c|c|c|c|c|c|c|c|c|c|c|}
\hline 2008 & 1.104 & 1.065 & 1.144 & $<.0001$ & 0.209 & 0.004 & $<.0001$ & 0.016 & 0.013 & 0.2238 \\
\hline 2009 & 1.038 & 0.997 & 1.080 & 0.0710 & 0.192 & 0.004 & $<.0001$ & 0.000 & 0.013 & 0.9752 \\
\hline 2010 & 0.974 & 0.931 & 1.018 & 0.2418 & 0.176 & 0.004 & $<.0001$ & -0.073 & 0.013 & $<.0001$ \\
\hline 2011 & 0.736 & 0.695 & 0.779 & $<.0001$ & 0.163 & 0.004 & $<.0001$ & 0.079 & 0.013 & $<.0001$ \\
\hline 2012 & 0.483 & 0.445 & 0.523 & $<.0001$ & 0.157 & 0.004 & $<.0001$ & 0.045 & 0.013 & 0.0007 \\
\hline 2013 & 0.304 & 0.265 & 0.350 & $<.0001$ & 0.149 & 0.004 & $<.0001$ & 0.091 & 0.014 & $<.0001$ \\
\hline Pre-dental care costs (per 1,000 KRW) & & & & & 0.001 & 0.000 & $<.0001$ & 0.004 & 0.000 & $<.0001$ \\
\hline Number of pre-dental care & & & & & 0.183 & 0.004 & $<.0001$ & 0.309 & 0.009 & $<.0001$ \\
\hline \multicolumn{11}{|l|}{ Dentofacial anomalies } \\
\hline Yes & 21.614 & 17.417 & 26.821 & $<.0001$ & 1.925 & 0.022 & $<.0001$ & 8.832 & 0.037 & $<.0001$ \\
\hline No & 1.000 & - & - & - & Ref & - & - & Ref & - & - \\
\hline \multicolumn{11}{|c|}{ Disorders of toothe development and eruption } \\
\hline Yes & 16.465 & 15.392 & 17.614 & $<.0001$ & 1.563 & 0.008 & $<.0001$ & 8.348 & 0.012 & $<.0001$ \\
\hline No & 1.000 & - & - & - & Ref & - & - & Ref & - & - \\
\hline
\end{tabular}

${ }^{a}$ The survival analysis using cox proportional hazard models was applied to investigate the association with risk of dental care visits during study period

${ }^{b}$ The negative binomial regression using GEE models was performed for patients who had experienced a dental care visit at least once per year to investigate the associations with dental care visits

${ }^{\mathrm{C}}$ The regression using GEE models was performed for patients who had experienced a dental care visit at least once per year to investigate the associations with dental care cost

programs since 1981 [12]. Previous studies of the water fluoridation programs showed that the programs had a positive role in preventing dental caries and improving oral health $[10,17,18]$. Based on those results, the water fluoridation programs seemed to gradually expand on the whole in South Korea. However, in the late 20th century, some concerns related to negative effects of water fluoridation, such as fluoride toxicity, were raised and proliferated. After that, the activation of the programs faced difficulties such as a negative public awareness. However, the previous studies of the adverse effects of water fluoridation found no significant adverse effects related to fluoride $[7,8,13]$. There was a need to change the public perception and provide evidence supporting the activation of water fluoridation programs for effective public health. Therefore, we examined the relationship between the implementation of water fluoridation and dental care utilization by South Koreans using a national sample cohort during 2003-2013.

Our findings showed that the implementation of the water fluoridation program in each region had a preventive role, decreasing dental care utilizations such as dental visits and expenditures. In the regions with water fluoridation programs, the duration of the program had an inverse association with the number of dental visits per year. Those findings were similar to the findings of previous studies including results in the other countries [8-11]. Water fluoridation programs as a prevention strategy could positively affect the improvement of oral health, and individuals who lived in regions with water fluoridation had less need to access dental care because of their improved oral health. The out-ofpocket expenditures for dental care in South Korea were remarkably higher than those in other OECD countries; introducing such prevention strategies had a quite positive affect on the reduction of the disease burden in South Korea [15]. Therefore, the implementation of water fluoridation improved oral health in South Korea, and a national recommendation for the activation of this program would be worthwhile based on our findings.

In addition, our study suggests some interesting results related to other covariates. The results for the financial independence rate of the local government as an indirect indicator of regional wealth revealed some inequalities in oral health among regions based on wealth, even if some community oral health programs were already implemented [19]. Hence, governments need to consider public health programs, such as regular dental screening programs, which could reduce inequalities. Also, the patients with a lower economic level, represented by income or type of insurance coverage, had a lower risk of dental visits and expenditures. Such results might be caused by the accessibility of health care. The wealthier individuals made more dental visits in the early stages of their clinical symptoms, and they used regular screening or consultation as part of primary and secondary prevention. On the other hand, individuals with low economic status could not make dental visits in the early stages of their symptoms and could not engage in prevention behaviors because of the cost burden [20, 21] Therefore, the makers of health policy such as Korea 
Table 4 The results of subgroup analysis for survival analysis, negative binomial regression, and regression by pre-dental care and patient age

\begin{tabular}{|c|c|c|c|c|c|c|c|c|c|c|c|c|}
\hline \multirow[t]{3}{*}{ Sub group } & & \multirow[t]{3}{*}{ Variables } & \multirow{2}{*}{\multicolumn{4}{|c|}{ Dental visits $^{a}$}} & \multicolumn{6}{|c|}{ Only patient with dental care visit } \\
\hline & & & & & & & \multicolumn{3}{|c|}{ Number of dental care visits ${ }^{\mathrm{b}}$} & \multicolumn{3}{|c|}{ Dental care costs $(\mathrm{KRW})^{\mathrm{C}}$} \\
\hline & & & $\overline{\mathrm{HR}}$ & $95 \% \mathrm{C}$ & & $P$-value & $\beta$ & SE & $P$-value & $\bar{\beta}$ & SE & $P$-value \\
\hline \multirow{4}{*}{$\begin{array}{l}\text { Experience of pre-dental care } \\
\text { in previous year }\end{array}$} & With & Water fluoridation & & & & & 0.043 & 0.041 & 0.2901 & 0.064 & 0.047 & 0.1712 \\
\hline & & $\begin{array}{l}\text { Period from implementation } \\
\text { of water fluoridation }\end{array}$ & & & & & -0.007 & 0.004 & 0.0689 & -0.003 & 0.004 & 0.4521 \\
\hline & Without & Water fluoridation & & & & & -0.031 & 0.015 & 0.0424 & -0.009 & 0.018 & 0.6180 \\
\hline & & $\begin{array}{l}\text { Period from implementation } \\
\text { of water fluoridation }\end{array}$ & & & & & -0.002 & 0.001 & 0.0959 & -0.004 & 0.002 & 0.0115 \\
\hline \multirow[t]{6}{*}{ Income (percentile) } & $-50 \%$ & Water fluoridation & 0.934 & 0.900 & 0.968 & 0.0002 & -0.0532 & 0.0247 & 0.0315 & -0.0106 & 0.0288 & 0.7131 \\
\hline & & $\begin{array}{l}\text { Period from implementation } \\
\text { of water fluoridation }\end{array}$ & 1.001 & 0.996 & 1.006 & 0.6484 & -0.0004 & 0.0022 & 0.8424 & -0.0026 & 0.0025 & 0.2927 \\
\hline & $51 \%$ & Water fluoridation & 0.957 & 0.930 & 0.985 & 0.0032 & -0.0083 & 0.0178 & 0.6422 & 0.0173 & 0.0212 & 0.4162 \\
\hline & & $\begin{array}{l}\text { Period from implementation } \\
\text { of water fluoridation }\end{array}$ & 0.997 & 0.993 & 1.000 & 0.0792 & -0.0050 & 0.0016 & 0.0014 & -0.0056 & 0.0018 & 0.0020 \\
\hline & -19 & Water fluoridation & 0.941 & 0.906 & 0.976 & 0.0013 & -0.0086 & 0.0232 & 0.7117 & 0.0048 & 0.0276 & 0.8617 \\
\hline & & $\begin{array}{l}\text { Period from implementation } \\
\text { of water fluoridation }\end{array}$ & 0.996 & 0.992 & 1.001 & 0.1132 & -0.0070 & 0.0021 & 0.0007 & -0.0054 & 0.0025 & 0.0270 \\
\hline \multirow[t]{6}{*}{ Age group } & $20-39$ & Water fluoridation & 0.959 & 0.922 & 0.998 & 0.0408 & -0.0113 & 0.0263 & 0.6675 & 0.0274 & 0.0309 & 0.3755 \\
\hline & & $\begin{array}{l}\text { Period from implementation } \\
\text { of water fluoridation }\end{array}$ & 1.001 & 0.996 & 1.006 & 0.8145 & -0.0034 & 0.0023 & 0.1495 & -0.0068 & 0.0027 & 0.0104 \\
\hline & $40-59$ & Water fluoridation & 0.952 & 0.908 & 0.999 & 0.0461 & -0.0568 & 0.0301 & 0.0595 & -0.0211 & 0.0352 & 0.5493 \\
\hline & & $\begin{array}{l}\text { Period from implementation } \\
\text { of water fluoridation }\end{array}$ & 1.000 & 0.994 & 1.006 & 0.9847 & -0.0001 & 0.0026 & 0.9676 & -0.0025 & 0.0029 & 0.3957 \\
\hline & $60+$ & Water fluoridation & 0.912 & 0.840 & 0.991 & 0.0297 & -0.0518 & 0.0465 & 0.2648 & 0.0010 & 0.0568 & 0.9864 \\
\hline & & $\begin{array}{l}\text { Period from implementation } \\
\text { of water fluoridation }\end{array}$ & 0.998 & 0.987 & 1.010 & 0.7551 & 0.0022 & 0.0039 & 0.5666 & 0.0008 & 0.0048 & 0.8707 \\
\hline
\end{tabular}

The reference level of water fluoridation was that of regions without a program, and the period since the implementation of water fluoridation was a continuous variable

The bold face indicates statistically significant results

These were results of sub group analyses for survival analysis and negative binomial regression analyses

${ }^{\text {aTT }}$ The survival analysis using cox proportional hazard models was applied to investigate the association with the risk of dental care visits

${ }^{\text {b}}$ The negative binomial regression using GEE model was performed for patients who had experienced a dental care visit at least once per year to investigate the associations with dental care visits.

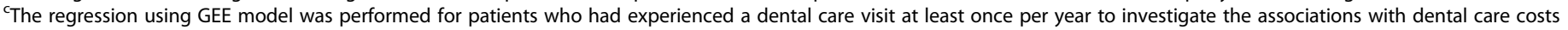


Health Promotion Foundation must consider some interventions to reduce economic barriers for those with low economic status to reduce health inequalities caused by economic issues. Finally, there were results showing that younger patients or patients with comorbidities utilized more dental care. Those results were similar to previous results about dental care. That is because younger patients are at a period of change from primary teeth to permanent teeth, whereas the elderly had fewer teeth because of deformation [22, 23]. Also, the patients with comorbid conditions such as dentofacial anomalies or disorders of tooth development and eruption made more dental visits than patients without those problems [24, 25]. Such patients need to activate preventive healthcare to reduce preventable expenditures.

The reduction of dental care utilization by the introduction of water fluoridation showed some differences in the subgroup analyses. The results showed that such associations were greater among patients that had not experienced dental care. It was suggested that the water fluoridation program had a major role in the reduction of the incidence of dental caries as a primary prevention rather than a secondary prevention. The implementation of such programs would be effective for individuals who managed their dental care well without clinical symptoms. Therefore, healthcare professionals need to recommend the importance of managing oral health even for individuals without any symptoms [26].

Our study has some strengths compared with previous studies. First, we used national health insurance national sampling cohort data to analyze the relationship between the implementation of water fluoridation programs and dental visits or expenditures. Therefore, the data used in our study are especially helpful for establishing evidence-based health policy for dental care. Second, to our best knowledge, this study is the first attempt to investigate the impact of water fluoridation programs on the whole nation of South Korea. So far, several previous studies of water fluoridation have been conducted, but most of those only investigated the impact of the programs at the community level [27-29]. Therefore, our findings would be effective evidence for establishing public health programs or policies. Third, we included the dental visits and expenditures as outcome variables. Therefore, our results could reflect overall dental care utilization by each patient. Finally, we adjusted the data for pre-dental care and other comorbid conditions to provide a more detailed study. Thus, we could reflect the severity of the dental caries experienced by the patients in our analyses.

Our study had also some limitations. Based on previous studies of dental caries or oral health, various factors including lifestyles, types of tooth paste, and frequencies of tooth brushing could affect the health outcome $[1,30]$. The impact of water fluoridation program could be different by cultural factors which affect to heathy behaviors in each countries. Also, the outcome variables in previous studies were used with other clinical indicators including the Patient Hygiene Performance index [31]. However, the relevant details were not included in the data, as the data used in our study had the characteristics of health insurance claim data. Therefore, we were unable to consider other variables that could affect dental caries. Next, we could not consider the fluoride level in the water or the actual consumption volume for each individual because of limitations of the data [32, 33]. In addition, we excluded the regions that quit the program during the study period, because we could not clearly identify the period effect after the introduction of water fluoridation. Therefore, more detailed studies are needed. Third, in the characteristics of dental care, there were many non-payment items related to dental care in South Korea. However, because of the characteristics of the NHI claim data, we could not consider those items. Thus, it is possible that the measurement of the dental care expenditures could have been underestimated. Finally, regarding some studies of water fluoridation programs that suggested that the programs had an adverse effect, there was a need to evaluate adverse effects. However, we only analyzed the dental care utilization and did not include any adverse effects.

Despite those limitations, our findings suggest that the implementation of water fluoridation programs had a positive role in improving oral health, and especially dental caries. Regarding the negative public perception of water fluoridation programs and the high out-of-pocket expenditures for dental care in South Korea, those findings could be helpful in the management of dental care expenditures from the perspective of health policy and public health. Although further detailed studies using clinical factors will be needed in the near future, makers of health policy and professionals in the area of dental care must consider efficient strategies for activating water fluoridation programs.

\section{Conclusions}

The implementation of water fluoridation programs is inversely associated with the risk of dental visits and expenditures related to dental caries. Considering that association, makers of health policy and decision makers such as Korea Health Promotion Foundation have to consider preventive strategies for activating water fluoridation programs, such as changes in public perception and relations, in order to effectively manage dental care in South Korea. 


\section{Appendix 1}

Table 5 Characteristics of study population at baseline

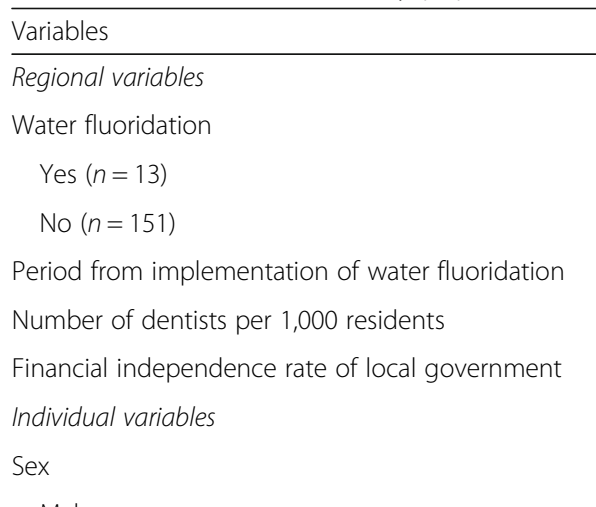

\section{Male}

Female

Age (Years)

-19
$20-29$
$30-39$
$40-49$
$50-59$
$60-69$
$>70$

Income (percentile)

$$
\begin{aligned}
& -10 \% \text { (low) } \\
& 11-20 \% \\
& 21-30 \% \\
& 31-40 \% \\
& 41-50 \% \\
& 51-60 \% \\
& 61-70 \% \\
& 71-80 \% \\
& 81-90 \% \\
& +91 \% \text { (high) }
\end{aligned}
$$

Types of insurance coverage

$$
\text { Medical Aid }
$$

$\mathrm{NHI}$, self-employed insured

$\mathrm{NHI}$, employee insured

Year of baseline

2003

2004

2005

2006

2007

2008

2009

2010
N/Mean

49,122

423,128

0.68

0.28

50.63

239,445

232,805

142,793

66,863

80,042

72,654

42,027

38,745

29,126

$49,834 \quad 10.55$

$30,220 \quad 6.40$

$34,226 \quad 7.25$

$40,371 \quad 8.55$

$45,727 \quad 9.68$

$48,872 \quad 10.35$

$54,782 \quad 11.60$

$58,121 \quad 12.31$

$58,827 \quad 12.46$

$51,270 \quad 10.86$

$\% / S D$

10.40

89.60

2.51

0.12

24.45

50.70

49.30

0.24

4.16

6.95

8.90

8.20

6.17

$18,607 \quad 3.94$

$213,931 \quad 45.30$

$239,712 \quad 50.76$

$412,110 \quad 87.27$

$11,234 \quad 2.38$

$6,703 \quad 1.42$

$5,847 \quad 1.24$

$5,576 \quad 1.18$

$5,156 \quad 1.09$

$4,838 \quad 1.02$
$6,440 \quad 1.36$
Table 5 Characteristics of study population at baseline (Continued)

\begin{tabular}{lll}
\hline 2011 & 4,567 & 0.97 \\
2012 & 4,918 & 1.04 \\
2013 & 4,861 & 1.03 \\
$\begin{array}{l}\text { Dentofacial anomalies } \\
\text { Yes }\end{array}$ & 83 & 0.02 \\
No & 472,167 & 99.98 \\
Disorders of toothe development and eruption & & \\
Yes & 881 & 0.19 \\
No & 471,369 & 99.81 \\
Follow up time (year) & 9.12 & 2.80 \\
Total & 472,250 & 100.00 \\
\hline
\end{tabular}

(1) 


\section{Appendix 2}



Fig. 1 The Kaplan-Meier Survival curve for time to first diagnosis of dental caries by the implementation water fluoridation

\section{Abbreviations}

Cl: Confidence interval; GEE: Generalized estimating equation; HR: Hazard ratio; ICD: International classification of diseases; KNHIS: Korean National Health Insurance Service; NHI: National Health Insurance; OECD: Organization for Economic Co-operation and Development; SE: Standard error; WHO: World Health Organization

\section{Acknowledgement}

No specific funding supported this study.

\section{Availability of data and materials}

The data used in this study were obtained from the NHIS National Sample Cohort, it can only be disclosed to the people who had authorized by NHIS. For obtaining the NHIS National Sampling Cohort, go to the following web site, and submit the application form (https://nhiss.nhis.or.kr/bd/ab/ bdaba021eng.do). The committee will evaluate that, and notice the determination of deliberation within 25 days from the data of application. And then, applicants who passed deliberation can use this data after payment of fee.

\section{Authors' contributions}

MSC and KTH designed the study, performed the research and statistical analyses, and wrote the manuscript. SHP, KTM, and ECP contributed to the discussion and reviewed and edited the manuscript. ECP is the guarantor of this work and as such has full access to all the data in the study and takes responsibility for the integrity of the data and the accuracy of the data analysis. The English in this document has been checked by at least two professional editors, both native English speakers. All authors read and approved the final manuscript.

\section{Competing interests}

The authors declare that they have no competing interests.
Consent for publication

Not applicable.

Ethics approval and consent to participate

The data used in this study included the information about dental care utilization of patients, and it was not included informed consents of each patient. This is because that the data was anonymized and not identified prior to analysis. This study was approved by the Institutional Review Board, Yonsei University Graduate School of Public Health (2014-239).

\section{Author details}

${ }^{1}$ Department of Health Policy and Management, Graduate School of Public Health, Yonsei University, Seoul, Republic of Korea. ${ }^{2}$ Division of Management \& Planning, Korea Health Promotion Foundation, Seoul, Republic of Korea. ${ }^{3}$ Department of Public Health, Graduate School, Yonsei University, Seoul, Republic of Korea. ${ }^{4}$ Institute of Health Services Research, Yonsei University College of Medicine, Seoul, Republic of Korea. ${ }^{5}$ Department of Biostatistics, Graduate School of Public Health, Yonsei University, Seoul, Republic of Korea. ${ }^{6}$ National Evidence-Based Healthcare Collaborating Agency, Seoul, Republic of Korea. ${ }^{7}$ Department of Preventive Medicine, Yonsei University College of Medicine, Seoul, Republic of Korea.

Received: 10 July 2016 Accepted: 26 October 2016

Published online: 08 November 2016

\section{References}

1. Selwitz RH, Ismail Al, Pitts NB. Dental caries. Lancet. 2007:369(9555):51-9.

2. Marinho VC, Higgins J, Logan S, Sheiham A. Fluoride toothpastes for preventing dental caries in children and adolescents. The Cochrane Libr. 2003.

3. Tinanoff N, Palmer CA. Dietary determinants of dental caries and dietary recommendations for preschool children. J Public Health Dent. 2000;60(3): 197-206.

4. Axelsson P, Lindhe J, Nyström B. On the prevention of caries and periodontal disease. J Clin Periodontol. 1991;18(3):182-9. 
5. Lee $\mathrm{HJ}$, Han DH: Exploring the determinants of secular decreases in dental caries among Korean children. Community Dent Oral Epidemiol. 2015;43(4):357-65.

6. Arnold Jr FA, Dean HT, Jay P, Knutson JW. Effect of fluoridated public water supplies on dental caries prevalence: tenth year of the Grand RapidsMuskegon study. Public Health Rep. 1956;71(7):652.

7. Petersen PE, Lennon MA. Effective use of fluorides for the prevention of dental caries in the 21 st century: the WHO approach. Community Dent Oral Epidemiol. 2004;32(5):319-21.

8. McDonagh MS, Whiting PF, Wilson PM, Sutton AJ, Chestnutt I, Cooper J, Misso K, Bradley M, Treasure E, Kleijnen J. Systematic review of water fluoridation. BMJ. 2000;321(7265):855-9.

9. Lee M, Dennison PJ. Water fluoridation and dental caries in 5-and 12-yearold children from Canterbury and Wellington. N Z Dent J. 2004;100(1):10-5.

10. Armfield JM. Community effectiveness of public water fluoridation in reducing children's dental disease. Washington, DC: Public Health Reports; 2010:655-64.

11. Kobayashi S, Kawasaki K, Takagi O, Nakamura M, Fujii N, Shinzato M, Maki Y, Takaesu Y. Caries experience in subjects18-22 years of age after 13 years' discontinued water fluoridation in Okinawa. Community Dent Oral Epidemiol. 1992;20(2):81-3.

12. DI P, Hs M, Horowitz AM, Gift HC, KI J, Ss S. Knowledge of and practices related to caries prevention among Koreans. J Public Health Dent. 1994; 54(4):205-10.

13. Parnell C, Whelton $\mathrm{H}, \mathrm{O}^{\prime}$ Mullane D. Water fluoridation. Eur Arch Paediatr Dent. 2009;10(3):141-8.

14. Prevalence of dental caries. http://kosis.kr/statHtml/statHtml.do?orgld= 117\&tblld=DT_11702_N116\&conn_path=12. Accessed 18 July 2016.

15. Organization for Economic Cooperation and Development. Health at a Glance 2013: OECD Indicators. 2013.

16. Zorn CJ: Generalized estimating equation models for correlated data. A review with applications. Am J Pol Sci. 2001;45(2):470-90.

17. Armfield JM, Spencer AJ, Roberts-Thomson KF, Plastow K. Water fluoridation and the association of sugar-sweetened beverage consumption and dental caries in Australian children. Am J Public Health. 2013;103(3):494-500.

18. Cobiac $L$, Vos T. Cost-effectiveness of extending the coverage of water supply fluoridation for the prevention of dental caries in Australia. Community Dent Oral Epidemiol. 2012;40(4):369-76.

19. Hobdell M, Petersen PE, Clarkson J, Johnson N. Global goals for oral health 2020. Int Dent J. 2003;53(5):285-8.

20. Jeon JE, Chung WG, Kim NH. The reason of unmet dental need related socioeconomic status in Korea: Using the 4th Korea National Health and Nutritional Examination Survey. J Korean Acad Oral Health. 2012;36(1):73-81.

21. Swank ME, Vernon SW, Lairson DR. Patterns of preventive dental behavior. Public Health Rep. 1986:101(2):175.

22. Fure S, Zickert I. Incidence of tooth loss and dental caries in 60-, 70and 80-year-old Swedish individuals. Community Dent Oral Epidemiol. 1997;25(2):137-42.

23. Harris R, Nicoll AD, Adair PM, Pine CM. Risk factors for dental caries in young children: a systematic review of the literature. Community Dent Health. 2004;21(1):71-85.

24. Solorzano I, Salas M, Chavarría P, Beltrán-Aguilar E, Horowitz H. Prevalence and severity of dental caries in Costa Rican schoolchildren: results of the 1999 national survey. Int Dent J. 2005;55(1):24-30.

25. Barmes DE. Epidemiology of dental disease. J Clin Periodontol. 1977;4(5):80-93.

26. Wilkins EM. McCullough PA. Clinical practice of the dental hygienist. Philadelphia: Lea \& Febiger; 1983.

27. Cho HJ, Lee HS, Paik DI, Bae KH. Association of dental caries with socioeconomic status in relation to different water fluoridation levels. Community Dent Oral Epidemiol. 2014;42(6):536-42.

28. Kim M-K, Jung J-I, Kim M-J, Jun E-J, Kim H-N, Kim S-Y, Han D-H, Jeong S-H, Kim J-B. Cost-benefit analysis of a water fluoridation program for 11 years in Jinju, Korea. J Korean Acad Oral Health. 2014;38(2):118-28.

29. Cho HJ, Jin BH, Park DY, Jung SH, Lee HS, Paik DI, Bae KH. Systemic effect of water fluoridation on dental caries prevalence. Community Dent Oral Epidemiol. 2014;42(4):341-8.

30. Petersen PE. Sociobehavioural risk factors in dental caries-international perspectives. Community Dent Oral Epidemiol. 2005;33(4):274-9.
31. Ismail Al, Sohn W, Tellez M, Willem JM, Betz J, Lepkowski J. Risk indicators for dental caries using the International Caries Detection and Assessment System (ICDAS). Community Dent Oral Epidemiol. 2008:36(1):55-68.

32. Bassin EB, Wypij D, Davis RB, Mittleman MA. Age-specific fluoride exposure in drinking water and osteosarcoma (United States). Cancer Causes Control. 2006;17(4):421-8.

33. Pizzo G, Piscopo MR, Pizzo I, Giuliana G. Community water fluoridation and caries prevention: a critical review. Clin Oral Investig. 2007;11(3):189-93.

\section{Submit your next manuscript to BioMed Central and we will help you at every step:}

- We accept pre-submission inquiries

- Our selector tool helps you to find the most relevant journal

- We provide round the clock customer support

- Convenient online submission

- Thorough peer review

- Inclusion in PubMed and all major indexing services

- Maximum visibility for your research

Submit your manuscript at www.biomedcentral.com/submit
Biomed Central 